\title{
DELAYED FORMATION OF BULGES BY DYNAMICAL
}

\section{PROCESSES}

\author{
DANIEL PFENNIGER \\ Geneva Observatory, CH-1290 Sauverny, Switzerland
}

September 29, 1992

\begin{abstract}
Two mechanisms involving purely dynamical processes can lead to the formation of a bulge after its disc: 1) snall bulges $(1-2 \mathrm{kpc})$, including box-shaped bulges and mildly triaxial bulges, can result from the formation and destruction of a bar; 2 ) big bulges (> $2 \mathrm{kpc}$ ) à la Sombrero can grow following the accretion of small satellites. Fully consistent $N$-body simulations show that the fraction of galaxy mass accreted in this way needs to be larger than about $5 \%$. Less accretion does not create smaller bulges, but heats the whole disc. These dynamical effects transforming Hubble types from SB to SA and vice-versa over $\approx 1-2$ Gyr also indicate, by the secular growth of bulges, a general sense of galactic evolution from Sd to Sa.
\end{abstract}

Key words: Bulge formation - Galaxy evolution - N-body simulation

\section{Constraints from Observations and Models}

The existence of a large fraction of metal rich stars in the Galactic Bulge, discussed many times in the recent years (cf. Rich, this conference) suggests that the classical scenario of galaxy formation, in which bulges form before their disc, is not the unique possibility. Scenarios in which bulges grow from disc material have to be considered. Also a number of other facts concerning galaxies that were not clearly perceived before the 70's should now be taken into account:

1) Numerous bulgeless galaxies (Sm, Sd) do exist. The percentage of low surface brightness galaxies is probably underestimated due to sky light contamination. These galaxies are example of discs, sometimes extremely thin, able to form without bulge. Such discs should fit in the galaxy formation scenario. Late type galaxies typically lack of symmetry, contrary to early type ones. The earlier the galaxies, the more wound are the spiral arms, suggesting that late type galaxies are dynamically "young"; $N$-body models show that large scale irregularities imply rapid morphological changes with a time-scale of order of $0.2 \mathrm{Gyr}$. After several rotations an unpertubed disc increases its symmetry by winding up its spiral arms.

2) Small bulge galaxies also exist (Sc's). Such small bulges have a radius smaller than or similar to the disc scale-length. In several cases Sc bulges are bluish and rapidly rotating (Kormendy, this conference) suggesting a causal link with their disc. Yet small bulges can hardly be made by accretion after the formation of the disc without heating the disc to a similar "temperature". Indeed for such a scenario, falling in material needs not only to have an unrealistically small amount of initial angular momentum in order to hit directly the central region, but also to lose all its kinetic energy in less than half a crossing time. Otherwise at the subsequent rebounds the disc would inevitably be traversed and heated.

$3)$ Decades of $N$-body experiments show that galactic discs are extremely "live" objects. Small perturbations on collisionless discs, such as the flyby of Magellanic satellites $^{[1]}$, or the inclusion of gas effects ${ }^{[2]}$, often produce large evanescent spiral arms. Basically the disc fragility results from the $Q$ stability parameter being near 
to critical over a wide range of radii. Marginal stability can be maintained by a feedback mechanism between the general energy dissipation, due to gas cooling, and the heating due to massive star formation triggered by large scale gravitational instabilities ${ }^{[3]}$. Disc instabilities easily produce rotating bars. Also the gas consumption rate is often short. All these mechanisms are able to induce a secular evolution over a time-scale much shorter than $10 \mathrm{Gyr}$. Assuming steady galactic potentials over such long time-intervals now appears as unrealistic.

4) Galaxy catalogues show that truly axisymmetric disc galaxies constitute a minority $((\mathrm{SA}: \mathrm{SAB}: \mathrm{SB}) \approx(1: 1: 1))$. Non-axisymmetric deformations occur especially often within the bulge region. Furthermore, SA's include edge-on or dusty galaxies for which axisymmetry is assumed, but increasingly disproved by observations peering through dust (e.g. Zarinsky et al., this conference). Now, according to several studies about bar dynamics ${ }^{[4-5]}$, even small or weak bars induce effects far from negligible. Indeed bars imply the existence of numerous radial and vertical orbital resonances, especially inside corotation ${ }^{[6]}$. Therefore their interaction and their causal connection with bulges must be investigated.

\section{Formation of Small Bulges}

In recent years, two dynamical mechanisms able to build small bulges from a disc have been described in the literature. Both mechanisms require a bar.

A number of $N$-body studies in 3D have shown that dynamically cold discs at first produce, through a well documented global instability, a classical rotating bar. Then, for a wide range of initial conditions, a $2 / 1$ vertical orbital resonance in the bar triggers another collective bending instability perpendicular to the galactic plane $^{[7-11]}$, lifting stars moving close to the plane up to $1-2 \mathrm{kpc}$. The resulting bar is made of disc material and appears as a peanut-shaped bulge when the bar is seen edge-on, and as a round bulge when seen end-on. When no dissipative effect is included, this boxy structure is stable over more than $10 \mathrm{Gyr}^{[8]}$. Furthermore, insensitive to particular initial conditions, 1) the surrounding stellar disc tends toward an exponential shape due to the relaxation induced by the bar, and 2) the bar region develops a steeper nearly exponential bulge-like profile ${ }^{[12-13,8]}$.

Studies including small dissipative effects lead to considering the effect of condensing mass in the central regions of a bar. As the specific angular momentum is no longer conserved inside a bar, accretion proceeds much faster than in a disc. Furthermore a central mass concentration inside a bar always creates a number of radial and vertical resonances, in particular low order $2 / 1$ resonances. It turns out that in fully self-consistent models a mass concentration inside the ILR of $1-3 \%$ of the total stellar disc mass is sufficient to dissolve a strong bar ${ }^{[14]}$. Any process secularly accumulating mass near the centre leads to the dissolution of the bar ${ }^{[15-16]}$. Among the specifically studied mechanisms are artificial slow drag of a fraction of the $N$-body particles ${ }^{[14]}$, satellite accretion by dynamical friction ${ }^{[1]}$, and gas accretion by hydrodynamic SPH simulations ${ }^{[2]}$. The dissolving bar first takes a hotter and triaxial shape, and later on a spheroidal shape. This mechanism can therefore produce small round bulges made from disc material. In addition, the exponential disc and the steeper bulge profiles follow naturally. 


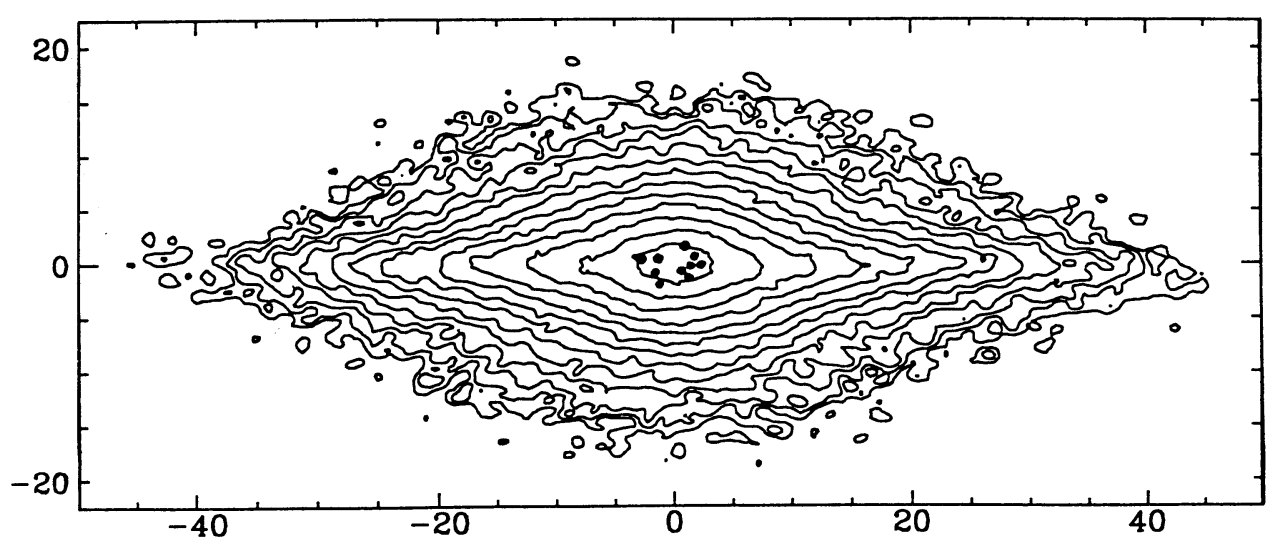

Fig. 1. Isophotes, in 0.5 mag steps, of the resulting Sombrero like disc when $f=10 \%$, seen edge-on. The ten satellites are marked by dots near the centre. The length unit is the kpc.

\section{Formation of Big Bulges}

The bulge class includes also big members, such as the Sombrero bulge. Such bulges, having a characteristic radius much larger than the disc scale-length can not result from disc material heated up in $z$ by internal instabilities such as bars, since the required energy exceeds the total mechanical energy that the disc and a possible bar can supply. But mergers falling in from large distances are an interesting source of large energy per unit mass. We know that equal sized mergers destroy discs and typically produce an ellipsoidal object ${ }^{[17]}$. Therefore it is natural to ask whether the cumulated accretion of small satellites can result in a galaxy type intermediate between a spiral and an elliptical, i.e. an early type spiral.

To quantify this scenario, we have performed a series of 3D collisionless PM $N$ body simulations with a polar grid and $N=2 \cdot 10^{5}$. Ten equal point mass satellites, without systematic rotation but supported by kinetic pressure start within a $30 \mathrm{kpc}$ radius sphere, and slowly accrete by dynamical friction toward the centre of a disc. The initial Miyamoto disc has a scale-length of $4 \mathrm{kpc}$ and a scale-height of $1 \mathrm{kpc}$, is in equilibrium, but is slightly bar unstable, as in [10]. Unperturbed it produces a peanut-shaped bulge-bar. The integration time amounts to $4 \mathrm{Gyr}$. The only varied parameter is the ratio $f$ of satellite mass to galaxy mass.

Below $f=2 \%$, the overall effect is negligible, also because dynamical friction is insufficient to drag the ten satellites close to the center within $4 \mathrm{Gyr}$. The peanutshaped bar is not significantly changed. Between $f=3 \%$ and $f=5 \%$, the satellites destroy the peanut shape of the bar, but not the bar itself; the bar becomes more oval. In this case, the persistence of the peanut shape traces the absence of merging events (and vice versa). Around $f=10 \%$, the bar is destroyed and the disc is significantly heated into a characteristic Sombrero like bulge with pointed isophotes along the disc (Fig. 1). Here all the mass in the big bulge comes from the disc. The bulge is hot and consequently rotates moderately. Above $f=10 \%$, the disc is more and more damaged and finally destroyed into an ellipsoidal object. 


\section{General Secular Evolution along the Hubble Sequence}

Since bulge formation is an irreversible process, if bulges form progressively after their disc, the direction of evolution along the disc Hubble sequence is from Sd to Sa. The recognition that galaxies can change of Hubble type in less than $10 \mathrm{Gyr}$ is not new. Equal sized mergers can make E galaxies ${ }^{[17]}$, and SB galaxies can be formed (in $\approx 0.2 \mathrm{Gyr}$ ) from SA galaxies spontaneously by the bar instability or induced by a satellite interaction ${ }^{[18,1]}$. We have described bar dissolving processes able to transform SB galaxies into SA ones with a larger bulge. A dissolving bar produces a bulge of similar size. Such a bar dissolution typically occurs as quickly as the accreting process is able to accumulate a critical mass within the bar ILR. Directly, repeated satellite accretion events tend to make only large bulges. Indirectly, satellites can boost central evolution by first inducing a bar by tidal interaction which can be eventually destroyed into a small bulge a few Gyr later.

The other properties of the Hubble sequence also suggest qualitatively that the sense of evolution is from $\mathrm{Sd}$ to $\mathrm{Sa}$. The rotation speed and surface luminosity increases can̉ result from the virial theorem applied to dissipative systems (present kinetic energy = dissipated mechanical energy). The degrees of symmetry and spiral arm opening can be understood by slow dynamical effects, because discs need several rotation periods to organize themselves. The gas fraction, the metallicity, and the colour can be attributed to the irreversible stellar activity processes.

In this picture the $\mathrm{Sm}$, Sd galaxies are closer to proto-galaxies than Sa's. Since late type galaxies are dynamically young, galaxies do not "form" necessarily only at an early brief epoch, but over an extended time interval, perhaps even up to now. Galaxy formation and galaxy evolution can not be separated, since late type galaxies can result from early type ones.

Several ideas in this work owe their origin to the influential lectures given by John Kormendy in the 1982 Saas Fee Course.

\section{References}

[1] Pfenniger, D.: 1991, in Dynamics of Disc Galaxies, ed. B. Sundelius, Göteborg, 191

[2] Friedli, D., Benz, W.: 1992, A.A. in press

[3] Kennicutt, R.C.: 1989, Ap.J. 344, 685

[4] Athanassoula, E., Bienaymé, O., Martinet, L., Pfenniger, D.: 1983, A.A. 127, 349

[5] Contopoulos, G., Papayannopoulos, Th.D.: 1980, A.A. 92, 33

[6] Pfenniger, D.: 1984, A.A. 134, 373, and 1985, A.A. 150, 112

[7] Combes, F., Sanders, R.H.: 1981, A.A. 96, 164

[8] Combes, F., Debbasch, F., Friedli, D., Pfenniger, D.: 1990, A.A. 233, 82

[9] Friedli, D., Pfenniger. D.: 1990, in Bulges of Galaxies, ESO Conference and Workshop Proceedings No. 35, eds. B.J. Jarvis, D.M. Terndrup, 265

[10] Pfenniger, D., Friedli, D.: 1991, A.A. 252, 75

[11] Raha, N., Sellw ood, .J.A., James, R.A., Kahn, F.D.: 1991, Nature 352, 411

[12] Hohl, F.: 1971, Ap.J. 168, 343

[13] Pfenniger, D.: 1990, A.A. 230, 55

[14] Friedli, D., Pfenniger, D.: 1991, in Dynamics of Galaxies and Molecular Clouds Distribution, IAU Symp. No 146, eds. Casoli F., Combes, F., Dordrecht: Kluwer, 362

[15] Hasan, H., Norman, C.: 1990, Ap.J. 361, 69

[16] Pfenniger, D., Norman, C.: 1990, Ap.J. 363, 391

[17] Barnes, J.E.: 1992, Ap.J. 393, 484

[18] Noguchi, M.: 1987, M.N.R.A.S. 228, 635 